Indonesian Journal of Physics

Vol 22 No. 4, October 2011

\title{
Dynamical Evolution of Sub-km Sized Main-belt Asteroids with Involving Thermal Radiation Effects
}

\author{
Budi Dermawan ${ }^{1}$ and A. Fermita ${ }^{2}$ \\ ${ }^{1}$ Astronomy Research Division, Institut Teknologi Bandung \\ ${ }^{2}$ Master Program in Astronomy, Institut Teknologi Bandung \\ e-mail: budider@as.itb.ac.id
}

Received 11 July 2011, Revised 14 August 2011, Accepted 23 September 2011

\begin{abstract}
Inconsistencies between asteroid's evolutionary model and observational results have been convincingly explained by thermal radiation effects that cause asteroids to experience orbital drifts. To investigate influences of the effects, we conducted 10 million yr orbital integration of hypothetical sub-km sized Main-belt asteroids. Numerical integration was run to propagate orbital dynamics of the asteroids by taking into account typical physical parameters of regolith- and basaltic-type surfaces of asteroids. Delivery processes from the Main-belt asteroids to near-Earth space are occurred by the aids of planetary orbital resonances and gravitational perturbations. In general, we find that thermal radiation effects play as an important role in the early evolution $(<1$ million yr), which guide the asteroids to follow different evolutionary paths than those of without involving the effects. This study indicates that asteroids whose surface belongs to basaltic-type have higher orbital mobility.
\end{abstract}

Keywords: Main-belt asteroids;Tthermal radiation effects.

\section{Introduction}

In the last decade it has been undeniably believed that inner and central Main-belt Asteroids (MBAs) are main sources of near-Earth Asteroids (NEAs). Along evolutionary tracks arriving at nearEarth space, the asteroids have been experienced some mechanisms before some of them are ejected out from the Main-belt. Classical evolutionary model, comprising only collisions and gravitational perturbations among asteroids in the Main-belt, fails to explain recent observational results of NEAs and meteorites $^{1)}$.

To explain the inconsistencies, a new mechanism has been proposed that belongs to a new type of non-gravitational force acting on a small body. Previously, the solar radiation pressure, the PoyntingRobertson effect, and the cometary outgassing have been categorized as types of non-gravitational forces. The new mechanism is thermal radiation coming out from asteroid's surface stirred up by solar radiation. The thermal radiation emerges forces and torques on a non spherical-shape asteroid, of which are very small compared to gravity but larger than the first-two nongravitational forces known above. However, for million-year-scale evolution this new mechanism plays as an important responsibility in orbital dynamics of asteroids that goes together with, surely, the gravity. This duo convincingly explains NEAs and meteorites originated from MBAs ${ }^{1)}$.

The thermal effects ${ }^{1)}$ include Yarkovsky radiation forces and YORP torques that cause $\sim 10-\mathrm{cm}$ to tens-km asteroids to drift the semi-major axis $(a)$, pump the eccentricity $(e)$ and the inclination (i) for a time scale of several million yr, and modify their spin vectors $^{2}$. Thermal radiations occur when a net thermal force (thermal absorption - emission) on a body is not zero. The thermal forces consist of two components, i.e. diurnal and seasonal. For diurnal component, a fraction of the solar insolation on an asteroid is absorbed only to later be radiated away, yielding a net thermal force in the direction that may cause the asteroid to spiral-outward or spiral-inward regarding its prograde or retrograde rotation, respectively. Diurnal component takes place on $100 \mathrm{~m}$ to $10 \mathrm{~km}$ objects having low thermal conductivity. While for seasonal component, the seasonal heating and cooling of the northern and southern hemispheres give rise of a thermal force, which lies along the spin axis. The net effect over one revolution always causes the asteroids to spiral-inward. Seasonal component occurs on smaller asteroids with sizes of 1 to $100 \mathrm{~m}$.

The YORP torques modify asteroid's spin rates and obliquities. This torque can spin-up and spindown rotation period of asteroids. The main reason is the fact that most asteroids have irregular shapes that the reflection and reemission of sunlight from an asteroid's surface can produce a net thermal torque. Over time, the torques can affect the spin rate and obliquity of the asteroid. Asteroid's shape, size, orbital distance, and spin-state are properties affecting YORP torques. Detailed description and mathematical formulations of the thermal radiation forces and torques are given elsewhere ${ }^{1,3)}$.

One important aspect of the effects that motivates this study is action of the effects in the delivery of sub-km-sized asteroids from their source locations in the Main-belt to near-Earth space through some mean-motion orbital resonances located in the inner region of Main-belt and the planetary perturbations. In this study, the thermal radiation forces play a major part of the effects rather than the torques for asteroid's sizes of about several hundred meters. A previous numerical study of MBAs 
evolution $^{2)}$ presumed mainly non-basaltic-type surface of the asteroids. Recent studies ${ }^{4,5)}$ show that it has become clear that appropriate number of basaltic-type asteroids occupy inner MBAs that belong to Vesta family and Vestoids. Basaltic-type surface is known to have thermal conductivity much higher than that of regolith-type one ${ }^{6)}$. Here in this study it is natural for us to include an appropriate fraction of basaltic-type surface asteroids and inspect their orbital evolutions.

\section{Orbital Evolution Scheme}

We conducted orbital integrations up to $10 \mathrm{Myr}$ by taking into account the thermal radiation effects. Two hundred hypothetical asteroids with sizes of 500 m were randomly generated between 2.1 to $2.8 \mathrm{AU}$, which represent samples of real MBAs in the inner (2.1 - 2.48 AU) and central (2.52 - $2.8 \mathrm{AU})$ regions. The inner and central regions of MBAs are separated by 3:1 mean-motion resonance of Jupiter (labeled by $3: 1 \mathrm{~J})$. Initial $a-e$ and $a-i$ random distributions of the asteroids are shown in Figure 1. Only major meanmotion resonances of terrestrial planets are drawn.

Following the previous work $^{7)}$ the SWIFT software package ${ }^{8)}$ was used to calculate and propagate evolutionary orbits of the asteroids by using regularized symplectic method with involving thermal radiation effects ${ }^{9}$. We used typical physical parameters of two types of asteroid's surface ${ }^{1)}$ listed in Table 1 with the same fraction number for the two types. Inner and central regions of MBAs are mainly populated by silicate and carbonaceous taxonomic types with albedos of 0.2 and 0.05 , respectively ${ }^{10)}$. We set 100 asteroids for each of the inner and central region of MBAs and spin vectors of all the asteroids are randomly generated.
Table 1. Physical properties of asteroids.

\begin{tabular}{lccc}
\hline Surface & $\begin{array}{c}\rho \\
\left(\mathrm{kg} / \mathrm{m}^{3}\right)\end{array}$ & $\begin{array}{c}\kappa \\
(\mathrm{W} / \mathrm{m} / \mathrm{K})\end{array}$ & $\begin{array}{c}C \\
(\mathrm{~J} / \mathrm{kg} / \mathrm{K})\end{array}$ \\
\hline \hline Basalt & 3500 & 2.659 & 680 \\
Regolith & 1500 & 0.0015 & 680 \\
\hline
\end{tabular}

$\rho$ is the density, $K$ the thermal conductivity, and $C$ the thermal capacity.

Dynamical evolution of the asteroids was represented by inspecting positions and velocities of the asteroids and all planets. This was done by employing numerical integration of general Cartesian N-body Newtonian's equations of motion along the time-evolution $^{11)}$. The asteroids are assumed to be massless. By taking an integration time-step of 80 days, we yielded that relative accuracies of the integration attain maximum of an order of $10^{-6}$, which is appropriate enough for a conservative system.

\section{Results and Discussion}

\subsection{General result}

We find that the thermal radiation effects play as an important role in the early evolution $(<1 \mathrm{Myr})$, which guides the asteroids to take different evolutionary tracks than those of without involving the effects. In dynamical evolution of MBAs, thermal radiation effects are significant for a time-scale of more than several $10^{5} \mathrm{yr}$. Snapshots of 1,5 , and 10 Myr evolutions are given in Figure 2, which shows that dynamical results between the left panels and the right ones differ significantly. Number of asteroids

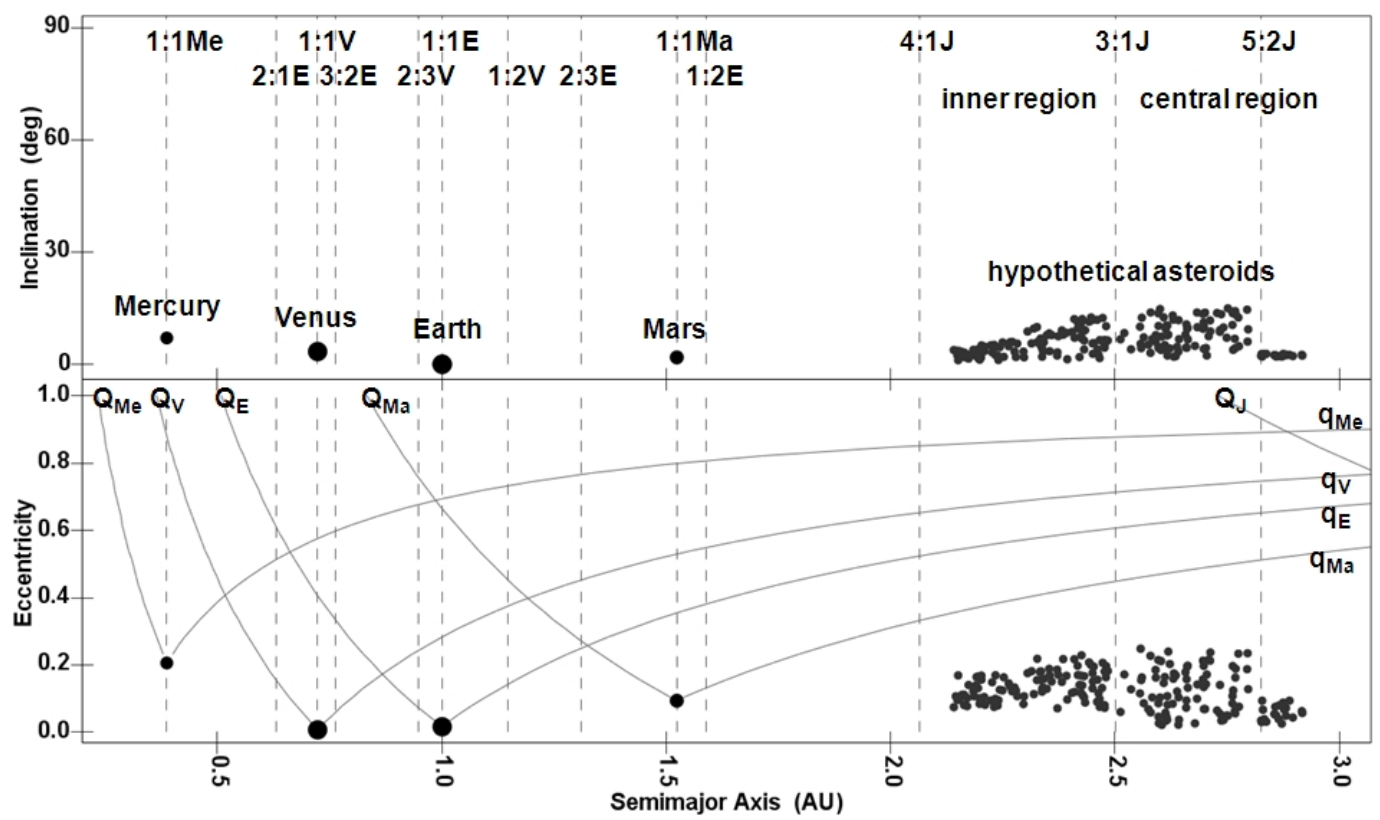

Figure 1. Initial orbital distributions of hypothetical asteroids residing in inner and central regions of the Main-belt. Vertical dashed lines stand for locations of planetary mean-motion resonances. Labels Me, V, E, Ma, and J denote Mercury, Venus, Earth, Mars, and Jupiter, respectively. Planetary aphelia (Q) and perihelia (q) are drawn by curves, respectively, on the left and the right to the corresponding planet. Marks and labels in this figure are also referred for all figures in this paper. 

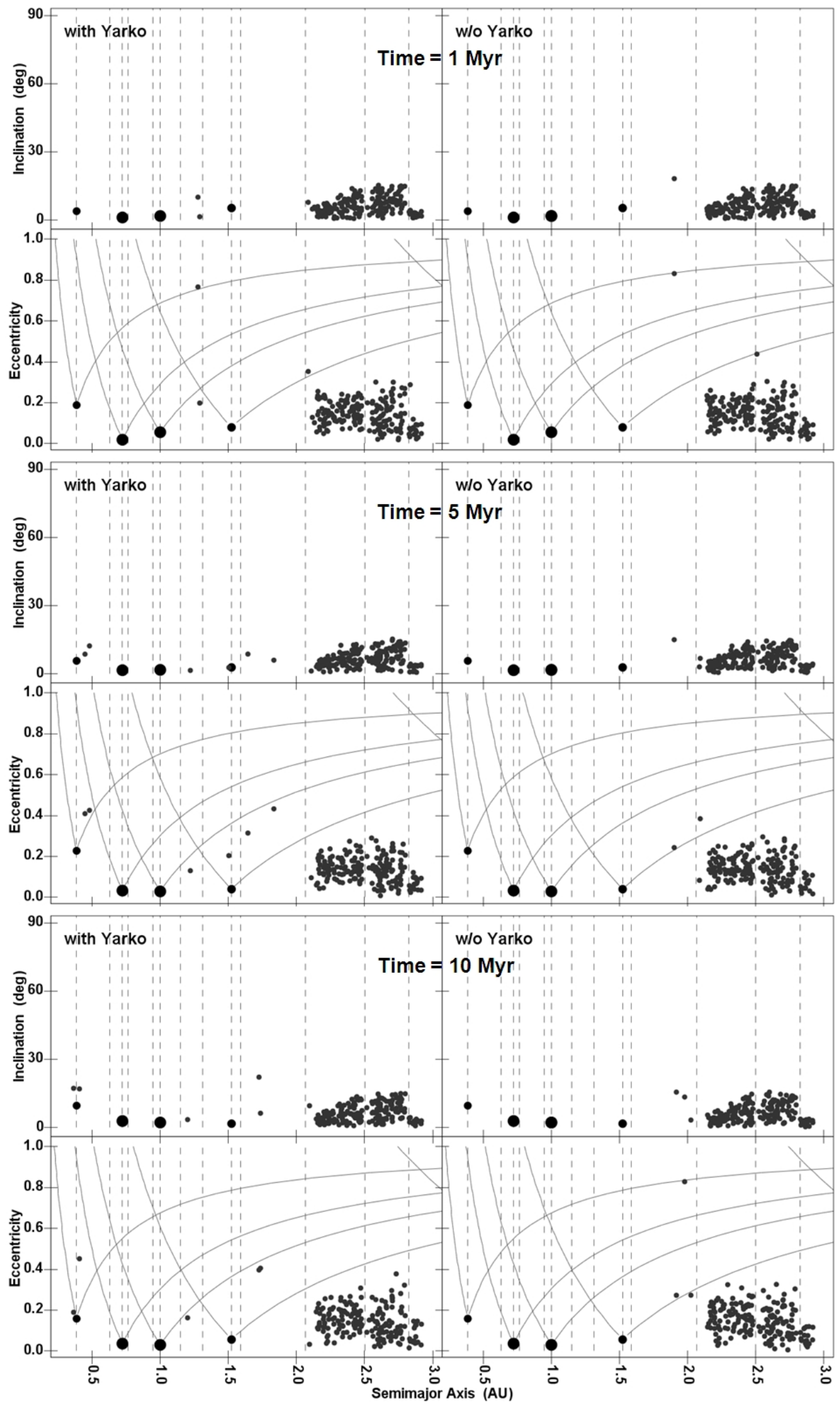

Figure 2. Snapshots of orbital evolutions of the hypothetical asteroids to near-Earth space for 1 (top), 5 (middle), and 10 (bottom) Myr. Left and right panels denote numerical integrations with and without thermal radiation effects, respectively. Marks in this figure are the same as those in Figure 1. 

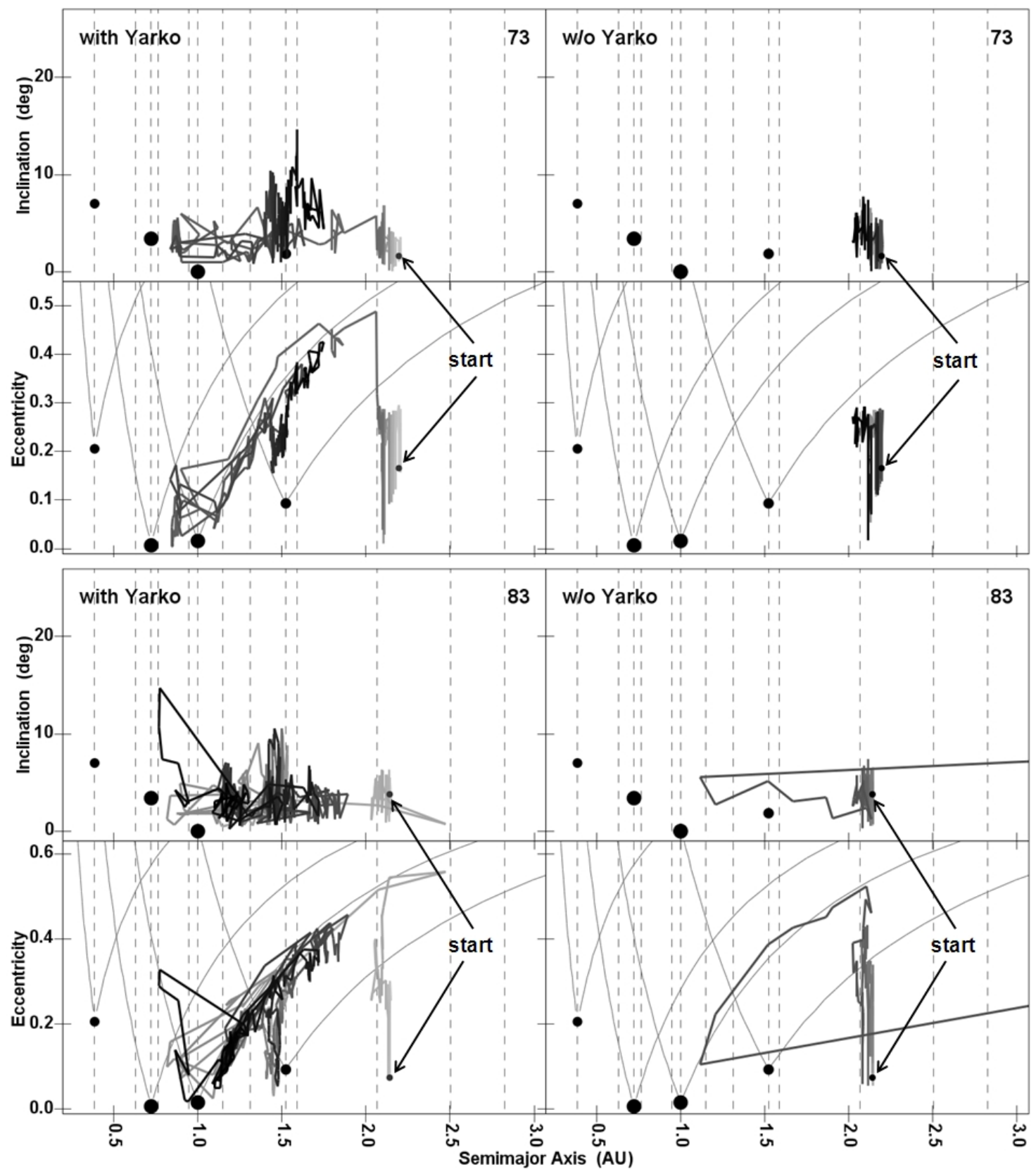

Figure 3. Dynamical evolution of two basaltic-type surface asteroids (73-top and 83-bottom panels) that occupy 4:1J mean-motion resonance. Time-evolution is denoted by gradual color-change from grey to black. Marks in this figure are the same as in Figure 1.

ejected out from the Main-belt entering near-Earth space is low for integrations without thermal effects (right panels). In the left panels, asteroids located near the mean-motion resonances suffer non-gravitational forces triggered by thermal radiation to drift their orbital elements entering the resonances. The asteroids have much higher possibility to be ejected out from the Main-belt to other locations in the solar system, especially to near-Earth space, when they stay shortly within the resonances. This provides as a plausible explanation that asteroids residing in near-Earth space, in the left panels of Figure 2, are more numerous than those of in the right panels.
Delivery processes of asteroids from the Mainbelt to near-Earth space are happened with aids of the planetary mean-motion resonances and planetary perturbations. Major mean-motion resonances engaged in this mechanism are 4:1J, 3:1J, and 5:2J, all with respect to Jupiter. They play as active "windows" for delivering the asteroids. The same fashion appears for the $v_{6}$ secular resonance with Saturn ${ }^{2)}$. Asteroids located far from the resonances have a very small chance to immediately escape from the Main-belt because they have to drift far enough to reach the resonances. Thus, they will be "old" asteroids when capable escaping from the Main-belt to near-Earth space. 
Comparing to the previous study of km-sized asteroids $^{7)}$, we find that the smaller the size, the higher the flux of asteroids is ejected out from the Main-belt. In this study of sub-km sized asteroids, we obtain considerable higher flux of asteroids coming out from the Main-belt to enter near-Earth and inner-Earth spaces. Furthermore, the ejected bodies from the Main-belt are dominated by basaltic-type surface asteroids. This indicates that mobility of basaltic-type surface of asteroids is higher than that of regolith-type surfaces.

\subsection{Samples of dynamical evolution}

Figure 3 shows two samples of evolutionary tracks of hypothetical asteroids 73 (top panels) and 83 (bottom panels) by utilizing the $4: 1 \mathrm{~J}$ mean-motion resonance. Both asteroids belong to basaltic-type surface. The thermal radiation effects shepherd elegantly the asteroids from the resonances to suit curves of planetary perturbations, and finally enter the near-Earth space to becoming Amors. This mechanism keeps number of NEAs population in a steady state behavior. This is one among important keys of thermal radiation effects that classical evolutionary model fails to answer. In the right panels, while asteroid 73 is relatively stable evolution, asteroid 83 shows rather similar evolution with its corresponding asteroid in the left panel for some several $10^{5} \mathrm{yr}$, before finally being catapulted to the outer solar system.

Figure 4 illustrates other two samples of evolution whose asteroids (145-top and 192-bottom panels) are ejected out by exploiting 3:1J and 5:2J mean-motion resonances. Final evolution of asteroid 145 (regolith-type surface) for both with and without thermal effects is same, i.e. in the outer solar system. However, their evolutionary tracks are different, particularly when terrestrial planetary perturbations are taken into account. Dynamics of asteroid 192 (basaltic-type surface) shows very divergent evolution between with and without thermal effects. By incorporating thermal effects, asteroid 192 may reach inner-Earth and even inner-Venus orbits (Atiras). Before entering Atira-type orbit, this asteroid is experienced with high inclination orbit when arriving at Apollo-type orbit.

\section{Conclusion}

Our results support what recently believe that, in general, mean-motion orbital resonances in the Main belt act as "windows" for delivering asteroids to other locations in the solar system. After being ejected out from the Main belt, asteroids will be perturbed by planets and their orbital elements change. During this process, thermal radiation effects play as a major key to guide the asteroids entering near-Earth space, particularly within time-evolution of less than $1 \mathrm{Myr}$.

Delivered asteroids into near-Earth space and the outer solar system are found to be numerous for basaltic-type. This is in consistent with new observational results that discover several tens basaltic-type asteroid residing in the inner region of MBAs and near-Earth space ${ }^{5}$. However, number of hypothetical asteroids in this study is much smaller than known asteroids in the Main-belt. Thus, it is reasonable to maintain an indication that asteroids whose surface belongs to basaltic-type have orbital mobility higher than those of regolith-type.

\section{Acknowledgement}

The authors thank Indonesian Journal of Physics (IJP) Internationalization Program in 2010 supported by Higher Education Directorate (Dikti) of Republic of Indonesia.

\section{References}

1. W. F. Bottke, et al., The Yarkovsky and YORP Effects, Ann. Rev. Earth Pla. Sci., 34, 157, 2006.

2. W. F. Bottke et al., Dynamical Evolution of Main Belt Meteoroids, Icarus, 145, 301, 2000.

3. E. Mysen, Dynamical Effects of Thermal Emission on Asteroids, Mon. Not. Royal Astron. Soc., 383, L50, 2008.

4. N. A. Moskovitz et al., The Distribution of Basaltic Asteroids in the Main Belt, Icarus, 198, 77, 2008.

5. R. Duffard, Basaltic Asteroids in the Solar System, Earth Moon Planet, 105, 221, 2009.

6. P. Farinella, D. Vokrouhlický, and W. K. Hartmann, Meteorite Delivery via Yarkovsky Orbital Drift, Icarus, 132, 378, 1998.

7. A. Fermita, Telaah Efek Radiasi Termal pada Asteroid, Final Project, Department of Astronomy, Institut Teknologi Bandung, 2007.

8. H. F. Levison and M. J. Duncan, The Long-term Dynamical Behavior of Short-period Comets, Icarus, 108, 18, 1994.

9. S. B. Bus and R. P. Binzel, Phase II of the Small Main-belt Asteroid Spectroscopic Survey, Icarus, 158, 146, 2002.

10. M. Brož et al., Non-gravitational Forces Acting on Small Bodies, in Proceedings of the 229th Symposium of IAU, Eds. L. Daniela, S. FerrazMello, \& F. J. Angel, Cambridge University Press, Cambridge, 351, 2006.

11. P. Bodenheimer et al., Numerical Methods in Astrophysics, Taylor \& Francis, New York, 2007. 